\title{
Smart home platform supporting decentralized adaptive automation control
}

\author{
Paolo Arcaini \\ National Institute of Informatics \\ Japan \\ arcaini@nii.ac.jp \\ Patrizia Scandurra \\ University of Bergamo, DIGIP \\ Italy \\ patrizia.scandurra@unibg.it
}

\author{
Raffaela Mirandola \\ Politecnico di Milano \\ Italy \\ raffaela.mirandola@polimi.it
}
Alberto Arrigoni
University of Bergamo, DIGIP
Italy
a.arrigoni5@studenti.unibg.it

\author{
Elvinia Riccobene \\ Università degli Studi di Milano, \\ Dipartimento di Informatica \\ Italy \\ elvinia.riccobene@unimi.it \\ Daniele Bosc \\ University of Bergamo, DIGIP \\ Italy \\ d.bosc@studenti.unibg.it
}

\author{
Federico Modica \\ University of Bergamo, DIGIP \\ Italy \\ f.modica@studenti.unibg.it
}

\author{
Rita Pedercini \\ University of Bergamo, DIGIP \\ Italy \\ r.pedercini@studenti.unibg.it
}

\begin{abstract}
Smart Home is an exemplar application domain of the Internet of Things (IoT). In particular, smart home automation processes that manage the interactions with the home devices and integrate all their (possibly interfering) services, introduce new challenges and call for modern engineering practices, software platforms, and computational intelligence. A key example is the need to achieve a more efficient integration between design and runtime aspects in the automation process, taking into account that unanticipated situations can occur in the home environment and require a smart home system to deal with them at runtime. In this paper, using an application example, we foresee how to architect future smart home systems in order to endow them with self-adaptation capabilities allowing decentralized control and device interconnection. Namely, we propose to exploit feedback control loop architectures (e.g., composite MAPE feedback loops) to design and manage the home automation processes, as alternative software architectural solutions to those of smart home platforms currently available.
\end{abstract}

\section{CCS CONCEPTS}

- Software and its engineering $\rightarrow$ Architecture description languages; Software design engineering.

\section{KEYWORDS}

Smart home platforms, software architecture styles for smart home systems, architecture-based self-adaptation, MAPE-K, OpenHAB

Permission to make digital or hard copies of all or part of this work for personal or classroom use is granted without fee provided that copies are not made or distributed for profit or commercial advantage and that copies bear this notice and the full citation on the first page. Copyrights for components of this work owned by others than ACM must be honored. Abstracting with credit is permitted. To copy otherwise, or republish, to post on servers or to redistribute to lists, requires prior specific permission and/or a fee. Request permissions from permissions@acm.org.

SAC '20, March 30-April 3, 2020, Brno, Czech Republic

(c) 2020 Association for Computing Machinery.

ACM ISBN 978-1-4503-6866-7/20/03...\$15.00

https://doi.org/10.1145/3341105.3373925

\section{ACM Reference Format:}

Paolo Arcaini, Raffaela Mirandola, Elvinia Riccobene, Patrizia Scandurra, Alberto Arrigoni, Daniele Bosc, Federico Modica, and Rita Pedercini. 2020. Smart home platform supporting decentralized adaptive automation control. In The 35th ACM/SIGAPP Symposium on Applied Computing (SAC '20), March 30-April 3, 2020, Brno, Czech Republic. ACM, New York, NY, USA, 8 pages. https://doi.org/10.1145/3341105.3373925

\section{INTRODUCTION}

The concept of Industry 4.0 has been recently adopted to refer to technologies like Cyber-Physical Systems, Internet of Things (IoT), Internet of Services, Internet of Energy that shall be used for the development of smart products. In this context, smart home systems constitute an exemplary situation where this concept of digital ecosystems can be seen at work. Besides, according to the IDC Mobile Device Trackers IDC's Worldwide Quarterly Smart Home Device Tracker $^{1}$, the smart home market, still in its infancy, is expected to grow considerably over the next few years in innovation and adoption. The general objective pursued in this context is that of connecting people to their home digital ecosystem made of things, software platforms, and value-added business services.

However, currently available smart home platforms need to be enhanced to scale up for real-life at home and to effectively provide significant benefits and human well-being at operation time. Currently, there is a lack of standardization w.r.t. hardware/software platforms for managing smart home processes, leading to a vast landscape of isolated, incompatible, task-specific and, thus, nonreusable solutions. Moreover, smart home automation processes, especially when managing the interactions with the home devices and integrating all their, possible interfering, offered services, introduce various new challenges and call for modern engineering practices, software platforms, and computational intelligence. To this extent, one of the major challenges is to deal with the design complexity of smart home automation processes that have to

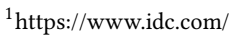


achieve a more efficient integration between the design and runtime aspects of the concerned systems. The emergence of new error sources and unanticipated situations within the home at run-time require a smart home system to be able to react dynamically to unforeseen situations. Consequently, there is a need for engineering practices, software platforms, and computational intelligence for the design, implementation, and execution of remote monitoring and control software for smart homes.

In this paper, we investigate how architecting future smart home systems by exploiting architecture-based self-adaptation. Concretely, we envisage an adaptive control architecture model made by multiple interacting Monitor-Analyze-Plan-Execute feedback loops over a shared Knowledge (MAPE-K)[11], called MAPE-K patterns, as alternative architectural style to those of smart home systems currently available on the market. The main idea is to endow current home automation platforms and applications with autonomic capabilities and decentralized control as required in an IoT context.

To demonstrate the technical feasibility of our approach, we adopt the open source platform for smart home installations OpenHAB (Open Home Automation Bus) ${ }^{2}$ and we show, using a smart home application example, how to encode the MAPE-K patterns to embed in the platform itself the self adaptation layer. To this end, we exploit the MSL (MAPE Specification Language) language and framework $[2,3]$ to model the adaptation logic of the system in terms of interacting MAPE-K patterns. Starting from MSL models, the corresponding encoding control rules for OpenHAB are then automatically derived. This mapping captures the execution semantics of the MAPE-K patterns, providing a fast and semantic preserving implementation of adaptive automation rules.

The main contributions of this paper are as follows:

- An adaptive control architecture style based on patterns of MAPE-K feedback loops for engineering smart home automation processes in terms of feedback controllers.

- An implementation of the adaptive control architecture style for OpenHAB-based smart home installations through an automated encoding of patterns instances of MAPE-K feedback loops into OpenHAB automation rules.

- A first validation of the architecture style in OpenHAB with automation scenarios of a smart home application example.

The rest of the paper is organized as follows. Sect. 2 illustrates our application domain, and Sect. 3 presents the envisioned architecturebased self-adaptation approach for smart home systems. An example of adaptive control architectures in the existing OpenHab platform is shown in Sect. 4. Finally, Sect. 5 discusses some related work, and Sect. 6 concludes the paper.

\section{THE CASE OF SMART HOME DOMAIN}

The adoption of smart everything and connected devices in our homes have facilitated the automation of a lot of tasks we previously had to take care of by ourselves. Optimizing energy consumption, providing comfortable heating based on our habits, controlling the watering of our plants when we are away, letting our fridge do the shopping by talking to our phone, etc., are becoming real use case scenarios in our home device ecosystem [7].

\footnotetext{
${ }^{2}$ https://www.openhab.org/
}

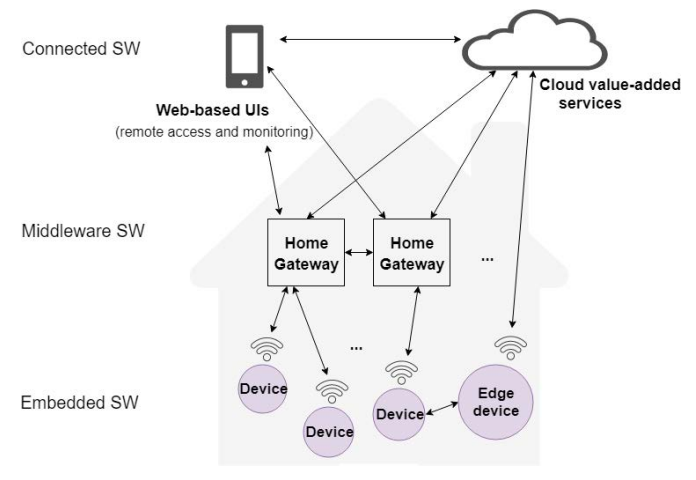

Figure 1: Smart home high-level architecture - Gatewayand Cloud-based integration

Important architectural requirements for a smart home system are dynamic integration of many types of drivers and devices, and interoperability with external (possibly remote) software applications/services by device manufacturers (to monitor and maintain devices remotely) or by third-parties providing value-added services (such as energy management and security surveillance). To achieve such requirements, current available smart home automation systems adopt a common architectural style (Gateway Integration Pattern) [12, 14, 18] where a residential gateway works as a mediator between a web or cloud application of a utility company and the smart home devices/appliances. These last are typically resource-constrained and do not directly connect to the Internet, and so the gateway bridges them to the web. Fig. 1 shows a highlevel deployment view of a typical smart home system's architecture using a free-style notation. The gateway collects raw and/or processed data from the home devices and forwards them to the remote applications and value-added cloud services; but, whenever possible, it also performs local data processing (computing in the edge) to reduce the data flow transmission. Moreover, a gateway can send commands to the smart devices, acting as a local scheduler or regulator to manage the control operations of the devices. So, a gateway supports a certain variety of local wireless communication protocols used by the devices (such as Z-Wave, ZigBee, WiFi, Bluetooth, etc.) and allows their interoperability, since generally resource-constrained devices cannot communicate with each other.

Multiple different gateways can be configured and integrated in one home, multi-gateway home installation, each supporting different local protocols and devices. There is the possibility for the user (human-in-the-loop) to control the system remotely through a simple UI application for smart-phones and tablets. When at home, users might also interact with the home devices (e.g., the thermostat, the smoke detector, or the video cameras) simply speaking in colloquial language. Amazon's Alexa and Google Assistant are examples of smart assistant platforms supporting such conversational AI. These very advanced solutions also employ AI techniques to identify and track users, learn the uses and habits of the home residents over time, so as to program their own control operations autonomously by inferred patterns of control.

In future scenarios involving edge devices with local computation capabilities, also direct device-to-device and device-to-cloud 
communication (Cloud Integration Pattern) [14] could be achieved without the gateway mediation (see the rightmost device depicted in Fig. 1). In this last scenario, Cloud services and apps would control numerous gateways and smart IoT devices over a wide geographic area to allow interoperability across IoT cloud platforms and application domains [18].

However, despite the recent advances in their conception, the design and development of a smart home control system is still very challenging. In general, it is difficult to design such control systems in a way that user well-being is guaranteed in every situation. Achieving one objective in isolation is fairly straightforward; achieving all objectives simultaneously is a complex task. Some real-life conflicts within the home environment can arise, for example, because unexpected events may happen or the home occupants may simultaneously be engaged in different activities with different and discordant preferences. The automation rules entered at design time or through a rule engine in operation time, or the inference patterns used by more intelligent solutions might be in conflict when more than one rule matches the same set of devices.

By exploiting MAPE-K feedback loops as general framework for autonomic computing, it is possible (as we envision in the next Section) to achieve a further evolution of this architectural style, currently mostly centralized and gateway-based, by conceiving the core software control in a decentralized setting. Moreover, recent results on reasoning about MAPE-K loops $[2,4]$ can help to analyze and arise conflicting situations.

\section{ARCHITECTURE-BASED SELF-ADAPTATION FOR SMART HOME AUTOMATION}

Smart home technology does not simply turn devices on and off; it can monitor the internal environment and the activities that are being undertaken by the occupants of the home and make decisions to configure the home's devices to their needs and living styles. In automating the home, a lot of devices interactions to perform collaborative tasks as desired by the home inhabitants can be autonomously performed without the human intervention. So we envision that, independently of the complexity of the control model, the structure of a smart home gateway can be expressed in terms of MAPE-K feedback loops dealing with different, interrelated, adaptation concerns of the (managed) smart home devices.

Hereafter, we first provide some background on MAPE-K control loops and their possible combination in terms of predefined patterns for self-adaptation; we then describe how an adaptive control architecture can be constructed from a set of these MAPE patterns, and how the behavior of a smart home system may be modeled, developed, tested, and understood.

\subsection{Architecture-based self-adaptation}

In architecture-based adaptation (see Fig. 2), the self-adaptation layer (the managing subsystem) is usually a set of interacting MAPEK (Monitor-Analyze-Plan-Execute over a Knowledge base) control loops [11], one per each adaptation concern. A component Knowledge $(\mathrm{K})$ maintains data of the managed system and environment, adaptation goals, and other relevant information shared by the MAPE components. A component Monitor (M) gathers data from

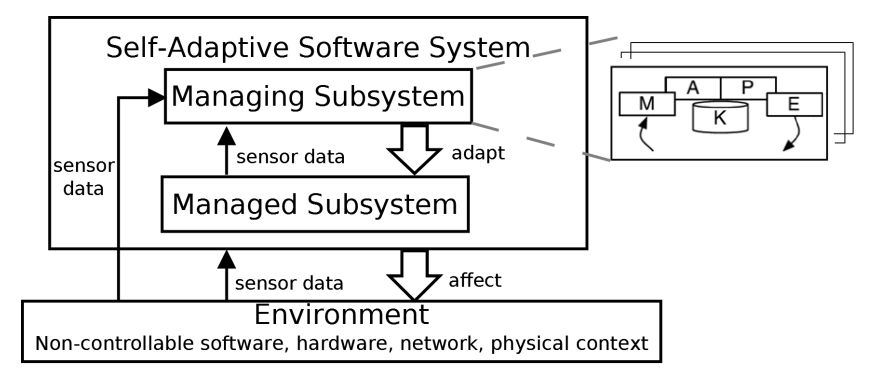

Figure 2: Architecture-based self-adaptation

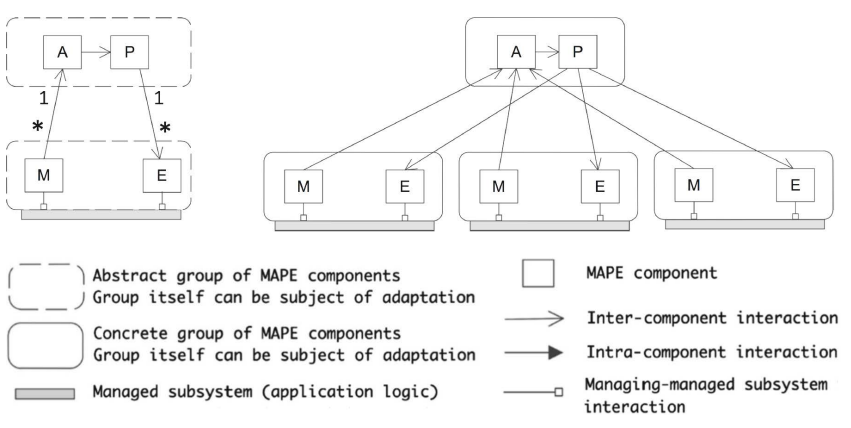

Figure 3: Pattern and instance of Master-slave MAPE (from [19])

the managed system and the environment through probes (or sensors), and saves data in the Knowledge. A component Analyze (A) performs data analysis to check whether an adaptation is required. If so, it triggers a component Plan (P) that composes a workflow of adaptation actions that are then carried out by a component Execution (E) through effectors (or actuators) of the managed system.

To deal with decentralized systems, some recurring structures of interacting MAPE components, MAPE patterns, have been defined [19] for designing decentralized adaptation solutions, where controllers make independent decisions but have some kind of interaction. Fig. 3 shows an example of MAPE pattern, the master-slave, an instance of this pattern in a configuration, and the key symbols of the graphical notation adopted in [19]. A MAPE pattern defines the structure of a composite MAPE loop as a set of abstract groups of MAPE components representing the roles of the feedback processes and the type of interactions between MAPE components. A pattern instance describes the structure of the pattern for one particular configuration. The annotated multiplicity of the interactions between the groups of MAPE components determines the allowed occurrences of the different groups in the pattern. Notationally, there are different types of interactions.

- Managing-managed subsystem interactions between M components and the managed subsystem for monitoring purposes, and between E components and the managed subsystem for performing adaptations.

- Inter-component interactions between different component types. Usually, $\mathrm{M}$ interacts with $\mathrm{A}, \mathrm{A}$ with $\mathrm{P}$, and $\mathrm{P}$ with $\mathrm{E}$.

- Intra-component interactions between components of the same type, e.g., interactions between $\mathrm{M}$ components. 


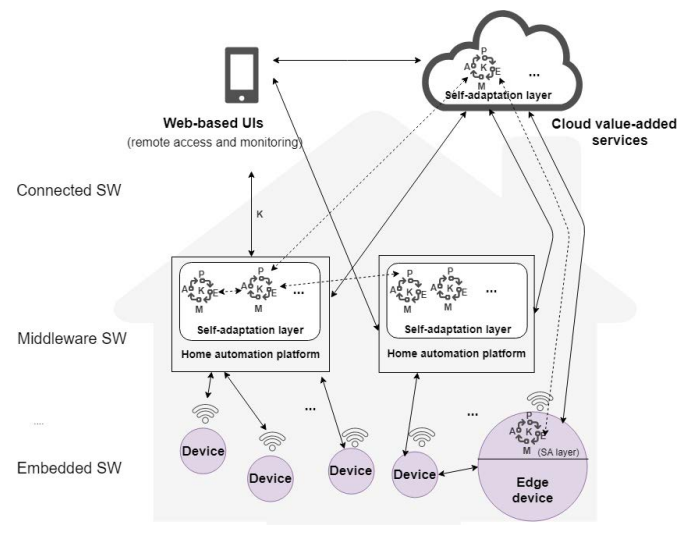

Figure 4: Vision overview of an adaptive control architecture for a smart home system

\subsection{Adaptive control architecture for smart home systems}

In order to reduce the design complexity of the home automation logic and also handle unexpected and uncertain operational conditions, the software control system of a smart environment may be architected as a self-adaptive system with two layers: the managed layer, for basic control operations, and a managing layer (or self-adaptation layer) realizing more advanced control operations through MAPE-K feedback loops. Fig. 4 provides a diagrammatic overview of the envisaged architecture.

The managed layer comprises the managed home devices and a software layer of the home automation platform providing hardware virtualization. This platform is a service-oriented component middleware that acts as an intermediate software layer between the home devices and software applications that make use of the data generated by the underlying technology, so providing device independence and hardware abstraction from specific home automation installations. Examples of complete open source, vendor- and technology- agnostic home automation platforms are, for example, OpenHAB, PiDome, HomeAssistant, Pimatic, Freedomotic. ${ }^{3}$ These home automation platforms are based on component frameworks and domain-specific modeling/coding languages to facilitate the design and development of a smart home automation system for a particular home installation. Precisely, these platforms play the role of middleware, since they provide virtualization and abstraction of the physical home devices, and allow remote user control apps (web or smartphones) to interact with the home devices through standard communication interfaces (e.g., REST APIs). Being hardware/protocol agnostic, in fact, they allow users to integrate and connect a variety of devices from classical home automation systems (such as KNX, Z-Wave, Insteon, EnOcean, etc.) to new IoT gadgets and devices (such as Koubachi, Sonos, Nest Labs, Philips Hue, GE Link and custom built Arduino nodes and sensors).

Additionally, some of these platforms (like OpenHAB) are endowed with experimental rule-based programming languages that allow the development, deployment, and execution of automation

${ }^{3}$ https://pidome.org/, https://home-assistant.io/, https://pimatic.org/, https://www. freedomotic-iot.com/ rules. Through these rule engines, users can manage several aspects of their smart home and create installation-specific automation rules or scenes dynamically. This feature allows using this platform as application container where several control aspects can be built and run anywhere (on a laptop, on a desktop computer, on test developer boards, on a Raspberry Pi, etc.). Therefore, the managing layer can be encoded directly in the rule engine of the platform.

The managing layer embedded in the home automation platform, is conceived as a set of multiple and interactive MAPE-K feedback loops, structured according to specific interaction patterns, dealing with several advanced control aspects (i.e., adaptation concerns). In a decentralized setting (e.g., for smart buildings, multi-floor homes, etc.), multiple groups of MAPE components interact to each other and are distributed over one or more instances of a home automation platform or over the edge-devices themselves (that would work as computational nodes) or on the Cloud, provided that an encoding of the MAPE-K loop execution semantics has been defined and implemented in the development board of the target computation platform. So, automation platforms may be used as execution containers for both the managed and the managing subsystems of an adaptive smart home automation system.

An important feature of a smart home system is its ability to make adaptation decisions, and this can be conceived on the base of different degrees of adaptivity [13]. The simplest one is that of a reactive control, but more advanced adaptive control models could be pursued such as those based on predicted activities (predictive control). Values that can be predicted include the device usage in the home, the movement patterns of the inhabitants and typical activities of the inhabitants. Proactive control could be also pursued by using the current available information to estimate what might happen next and adjust the system in advance. For these last advanced forms of adaptation (but also, in general, whenever the analysis of all adaptation options include formal analysis) computationally intensive reasoning is required and realized by the Analysis and Plan components of a MAPE loop with the help of complex analysis models (e.g., quality models, learning models, etc.) in the Knowledge repository and further components (verifiers, machine learners, etc.) $[8,10]$. These components are typically implemented as connected software/services running on the Cloud or on any other desktop platform, rather than in the home automation platform, since can be resource and time consuming.

\section{ADAPTIVE CONTROL ARCHITECTURES IN OPENHAB}

We here provide a concrete realization of the architecture model presented in the previous section for OpenHAB-based smart home installations. We choose OpenHAB because of its maturity level of development, widespread use, range of supported devices, and size of community. OpenHAB abstracts from the underlying devices protocols and adopts a Thing component model $l^{4}$ to represent and access the home environment devices via software components and APIs. So we rely on the OpenHAB concept of "binding" (a software adapter provided as add-on by the device manufacturer) that translates these high-level sensing/control commands in protocoldependent requests according to the specificity of the device.

\footnotetext{
${ }^{4}$ https://www.openhab.org/docs/concepts/
} 
In particular, through a running example, we describe how to encode MAPE-K loops of the self-adaptation layer of a smart home system into home automation processes of the OpenHAB platform. To this aim, we use the MSL (MAPE Specification Language) language [15] and design framework presented in $[2,3]$ to model the adaptation logic of a smart home system in terms of patterns of interactive MAPE-K loops. MSL allows the definition and instantiation of recurring structures of MAPE-K loops; in particular, it has been validated with the decentralized MAPE-K design patterns proposed in [19]. From an MSL model of a MAPE-K loop structure, a corresponding encoding in terms of control rules for the rule engine of OpenHAB can be automatically generated. This automatic mapping from MSL to OpenHAB captures the intended execution semantics of the MAPE patterns, so it provides fast and semanticspreserving implementation of home automation software designed with adaptation in mind. Moreover, thanks to the openness and dynamic modularization of OpenHAB (that is based on an OSGi architecture), MAPE loops for additional adaptation concerns can be introduced at any moment: they can be modelled in MSL, and then coded, refined, and deployed in OpenHAB at run-time.

\subsection{Running example: smart fire detection}

In order to keep the running example simple, we consider here a traditional view of control in the form of reactive adaptation: when an event occurs and causes the need for adaptation, an adaptation plan is carried out. We therefore do not consider the two further time perspectives of predictive adaptation and proactive adaptation that plan adaptation before an actual event happens.

We assume that a designer has to build an OpenHAB model of the home automation installation. So, to provide a proof-ofconcept evaluation and understand the benefits and limitations of our approach, we configured an OpenHAB instance for a two-floors (virtual) smart home ${ }^{5}$; each floor consists of rooms, and a room has several controlled devices. We conducted some experiments to model, implement and execute some home automation concerns in OpenHAB using our architecting approach for smart home systems.

We here describe a scenario concerning fire detection to achieve this adaptation goal: the system should detect and respond to the presence of a flame/smoke, allowing fire detection continuously. Being a domestic environment, flame/smoke sensors are used for fire sensing, and if smoke/flame is detected by these sensors, the smart home system should activate an alarm and/or send a notification to the user's smartphone. However, failed or silent flame/smoke sensors may undermine the successful execution of this basic fire detection and control process. To provide a more robust fire sensing, the fire sensing operation of a failed/silent fire sensor may be temporarily realized by the temperature sensors in the home ${ }^{6}$.

To this purpose, a MAPE-K loop is introduced into the managing layer of the smart home system to enable the triggering of the alarm even if none of the flame/smoke detectors switches to $\mathrm{ON}$ (as a consequence of sensor failure) but a room temperature sensor reports a temperature greater than a certain threshold (e.g., $45^{\circ} \mathrm{C}$ ). Code 1 reports the MSL definition of this MAPE-K loop as

\footnotetext{
${ }^{5}$ See the GitHub project https://github.com/scandurra/VirtualSmartHome-Project

${ }^{6}$ This policy is an example of re-allocation of functionality on differently capable devices, typically used in IoT scenarios to allow architecting emergent configurations [1] when some devices may be silent (or failed).
}

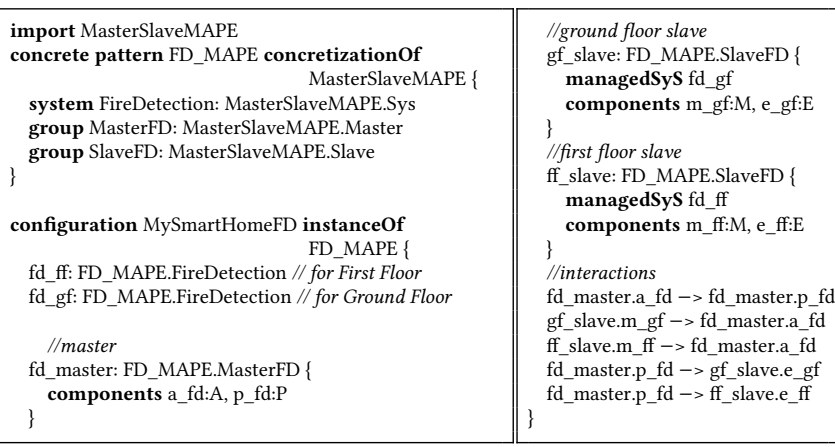

Code 1: MSL MAPE loop for guaranteeing fire detection

\begin{tabular}{|c|c|}
\hline $\begin{array}{l}\text { abstract pattern MasterSlaveMAPE \{ } \\
\text { system Sys } \\
\text { group Master \{ } \\
\text { components A, P } \\
\text { \} } \\
\text { group Slave \{ } \\
\text { managedSyS Sys } \\
\text { components M, E } \\
\text { \} }\end{array}$ & $\begin{array}{l}\text { interaction Slave.M } \rightarrow \text { Master.A }[*-\text { SOME, } 1] \\
\text { interaction Master.P } \rightarrow>\text { Slave.E }[1, *-\text { SOME }] \\
\text { interaction Master.A } \rightarrow \text { Master.P }[1,1] \\
\text { \} }\end{array}$ \\
\hline
\end{tabular}

Code 2: Master-slave MAPE pattern definition in MSL

instantiation of the Master-Slave MAPE pattern [19]. The two managed subsystems (fd_gf and fd_ff) take the form of fire alarm subsystems incorporating smoke and temperature sensors for the two floors, respectively: the ground floor (in the kitchen and living room), and the first floor for the bedrooms. Two slave components gf_slave and ff_slave are responsible for monitoring rooms temperature and activating alarms in the corresponding floors, while a (centralized) master component is responsible for the analysis and planning of proper actuation. For completeness, Code 2 shows the MSL definition of the Master-Slave MAPE pattern from [19].

Note that MSL allows only to model a compound structure of MAPE components and their interaction connectors, while the behavior of the MAPE components (how to monitor, how to analyze the knowledge, etc.) has to be specified by the developer using the implementation language of a target back-end platform integrated within the MSL framework. In our case, the corresponding encoding and behavior implementation in OpenHAB of the running MSL model example is presented in the next Subsection.

\subsection{Mapping patterns of interactive MAPE-K feedback loops into home automation rules}

A model compiler MSL2OpenHAB has been developed within the MSL framework to automatically translate an MSL model into an OpenHAB-based implementation. This MSL model compiler increases the use of OpenHAB as a platform to implement both the managed layer (comprising basic control functionalities and static automation modes/settings) and the managing layer for selfadaptation of a smart home system.

Essentially, from an MSL model of the structure of a composite MAPE loop, a set of OpenHab items ${ }^{7}$ (a file .items) and a set

\footnotetext{
${ }^{7}$ Items in OpenHAB represent capabilities of things (physical or virtual devices, web services or any other manageable source of information and functionality) in the managed home. Items have a state and they may receive commands.
} 
of Event-Condition-Actions rules (a file .rules) for the OpenHAB automation rule engine are generated. All rules generated in the rule file for an MSL model of a MAPE-K loop structure share a common execution context, i.e., they can directly access to all items of the underlying OpenHAB instance and also to variables (the knowledge of the MAPE groups) declared within the same rule file.

The basic execution semantics of the OpenHAB rule engine is that all rules (possibly defined in different rule files) are executed in parallel in reaction to one or more trigger conditions. OpenHAB supports different types of rule triggers: item- (or event-) based triggers, time-based triggers, etc. According to such a rule execution semantics and by exploiting the concept of item-based trigger of OpenHAB, we are able to encode in OpenHAB the MAPE-K execution semantics by enforcing a sequential triggering of the components of a MAPE group and by allowing components of different groups to trigger each other according to the interactions as established by the underlying MAPE pattern.

Table 1 summarizes, concept by concept, the mapping from MSL to the OpenHAB rule programming language. Basically, a concrete group $c g$ of MAPE components in an MSL model is mapped into an OpenHAB items group $c g$ in the item file. For each MAPE component instance $c i$ of type $X \in(M, A, P, E)$ of a group $c g$, a named OpenHAB rule $X_{-} c g_{-} c i$ and a Switch item start_cg_ci (that can carry $\mathrm{ON} / \mathrm{OFF}$ values) are generated. The rationale behind is that the switch item (when is $\mathrm{ON}$ ) is the triggering event upon which the rule logic (associated to the MAPE component $X$ ) is executed. As part of its action body, the rule $X$ is responsible for resetting to OFF its own triggering switch item.

In particular, a component instance $c i$ of type $A$ has a predefined template code since it is responsible for determining whether adaptation actions are required depending on the adaptation concern of interest and on the knowledge base about the state of the managed home devices and environment. To this purpose, a predicate adaptation_required_cg_a is introduced to represent the result of such analysis but its specification is application-specific so the designer/developer must supply an implementation for it. If it evaluates to true, the rule corresponding to the Plan component instance is triggered by setting to ON its triggering switch (start_cg_p in the table), otherwise the loop completes and the rule(s) corresponding to the Monitor component(s) of the loop are triggered by setting to ON its(their) triggering switch (start_cg_ $m$ in the table) to allow a next execution of the loop. Similarly, as indicator that the loop completed its current execution and to run through the next iteration of the loop, the rule body for a component instance $c i$ of type $E$ includes the triggering (the setting to $\mathrm{ON}$ of the switch start_cg_m in the table) of the rule corresponding to the Monitor component instance of the same concrete group $c g$.

A concrete interaction from a component instance ci1 to another component instance $c i 2$ of the same concrete group $c g$ or of different concrete groups $c g 1$ and $c g 2, c g 1 \neq c g 2$, is translated into a sendCommand for the OpenHAB event bus ${ }^{8}$ to set to ON the triggering switch item (start_cg_ci2 or start_cg2_ci2,respectively) of the rule associated to the target component instance ci2. This

\footnotetext{
${ }^{8}$ In case ci2 is deployed on a different OpenHAB platform instance, a command to its related items can be sent via the OpenHAB REST API or via an MQTT broker.
}

Table 1: Mapping from MSL models into OpenHAB

\begin{tabular}{|c|c|}
\hline MAPE element & OpenHAB construct \\
\hline Concrete group $\mathrm{cg}$ & Group cg "cg" \\
\hline $\begin{array}{l}\text { Component instance } \\
c i: X \in(M, A, P, E) \text { of } \\
\text { concrete group } c g\end{array}$ & $\begin{array}{l}\text { Switch start_cg_ci "ci" (cg) } \\
\text { rule "X_cg_ci" } \\
\text { when } \\
\quad \text { Item start_cg_ci received command ON } \\
\text { then } \\
\quad \text { sendCommand(start_cg_ci, OFF) } \\
\quad \quad \ldots \\
\text { end }\end{array}$ \\
\hline $\begin{array}{l}\text { Component instance } \\
a: A \text { of concrete group } \\
c g\end{array}$ & $\begin{array}{l}\text { rule "A_cg_a" } \\
\ldots \\
\text { then } \\
\ldots . \\
\text { if (adaptation_required_cg_a) } \\
\text { sendCommand(start_cg_p, ON) } \\
\text { else } \\
\quad \text { sendCommand(start_cg_m, ON) } \\
\text { end }\end{array}$ \\
\hline $\begin{array}{l}\text { Component instance } \\
e: E \text { of concrete group } \\
c g\end{array}$ & $\begin{array}{l}\text { rule "E_cg_e" } \\
\ldots \\
\text { then } \\
\ldots \\
\text { sendCommand(start_cg_m, ON) } \\
\text { end }\end{array}$ \\
\hline $\begin{array}{l}\text { Concrete interaction } \\
\text { cg.ci1 }->\text { cg.ci2 }\end{array}$ & $\begin{array}{l}\text { rule "X_cg_ci1" } \\
\quad \ldots \\
\text { then } \\
\quad \ldots \\
\text { sendCommand(start_cg_ci2, ON) } \\
\text { end }\end{array}$ \\
\hline $\begin{array}{l}\text { Concrete interaction } \\
\text { cg1.ci1 -> cg2.ci2 }\end{array}$ & $\begin{array}{l}\text { rule "X_cg1_ci1" } \\
\quad \ldots \\
\text { then } \\
\quad \ldots \\
\text { sendCommand(start_cg2_ci2, ON) } \\
\text { end }\end{array}$ \\
\hline $\begin{array}{l}\text { *-SOME semantics } \\
\text { cg1.ci1 }->\text { cg.ci } \\
\text { cg2.ci2 -> cg.ci }\end{array}$ & $\begin{array}{l}\text { Group:Switch:OR(ON, OFF) } \\
\qquad \text { cg1_cg2_to_cg "cg1_cg2_to_cg" } \\
\text { Switch cg1_to_cg "cg1_to_cg" (cg1_cg2_to_cg) } \\
\text { Switch cg2_to_cg "cg2_to_cg" (cg1_cg2_to_cg) }\end{array}$ \\
\hline $\begin{array}{l}\text { *-ALL semantics } \\
\text { cg1.ci1 -> cg.ci } \\
\text { cg2.ci2 -> cg.ci }\end{array}$ & $\begin{array}{l}\text { Group:Switch:AND(ON, OFF) } \\
\text { cg1_cg2_to_cg "cg1_cg2_to_cg" } \\
\text { Switch cg1_to_cg "cg1_to_cg" (cg1_cg2_to_cg) } \\
\text { Switch cg2_to_cg "cg2_to_cg" (cg1_cg2_to_cg) }\end{array}$ \\
\hline $\begin{array}{l}\text { * semantics } \\
\text { cg1.ci1 }->\text { cg.ci } \\
\text { cg2.ci2 }->\text { cg.ci }\end{array}$ & $\begin{array}{l}\text { rule "Aggregator_cg1_cg2_cg" } \\
\text { when } \\
\text { Item cg1_cg2_to_cg received update ON } \\
\text { then } \\
\begin{array}{l}\text { sendCommand(cg1_to_cg, OFF) } \\
\text { sendCommand(cg2_to_cg, OFF) }\end{array} \\
\text { sendCommand(start_cg_ci, ON) }\end{array}$ \\
\hline
\end{tabular}

command is part of the action body of the rule associated to the sender component instance $c i 1$. 


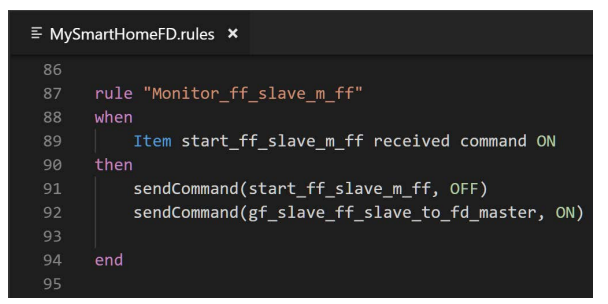

Figure 5: OpenHAB generated rule - Monitor_ff_slave_m_ff

The encoding supports interactions $\left[{ }^{*}, 1\right]$ from multiple components to one component of different MAPE groups with the starting multiplicity *-ALL and *-SOME, respectively meaning that the target group must receive the communication from all the inter-acting groups or from at least one of them. In OpenHAB, this triggering semantics is captured in terms of switches whose value is aggregated into a single status value of a group of item type Switch (groups cg1_cg2_to_cg in the table) using the aggregation function AND or OR, respectively. The state of such a group then represents the final state (i.e. whether all switches states are $\mathrm{ON}$ or at least one switch state is ON). In addition, a rule (rule Aggregator_cg1_cg2_cg in the table) is generated with the task of triggering the target component instance of the interaction $\left[{ }^{*}, 1\right]$ (by setting to ON its triggering switch) when the aggregate group of switches of the starter components is $\mathrm{ON}$, and resetting to OFF these last.

The rules definition obtained from an MSL model can be then manually refined to complete the automation logic (the MAPE components behavior) by specifying OpenHAB-specific actions on the items declared in the managed home description in OpenHAB. The rules file can then be placed into the rules directory of Open$\mathrm{HAB}$. Rule files for different adaptation/control concerns (different MAPE-K loops) will be generated; they all share the same Open$\mathrm{HAB}$ platform instance for a specific smart home (the managed subsystem and environment) and react on events on the OpenHAB event bus, i.e., commands and status updates for home devices.

Running example. The MAPE loop structure of the MSL model for the fire detection concern (see Code 1) can be coded automatically in OpenHAB to leverage the MAPE functionality to do concrete home automation. Fig. 5 shows, e.g., the rule generated from the $M$ component $m_{-} f f$ of the slave group $f f \_s l a v e$ for the fire sensing of the first floor. The Action block of this rule has been further refined to add application-specific control logic as shown in Fig. 6. Upon temperature sensors changing for the first floor, it saves into the knowledge (by variables cbTemp and bTemp) the temperature values of floor rooms (the children room and the bedroom).

The automation evolution (including the devices' state) of an OpenHAB instance configured for a specific smart home can be observed at runtime via the OpenHAB administration console or a dashboard accessible remotely (from a smartphone/tablet) using a web browser. This provides quickly a prototype of the managing subsystem (embedding the adaptation/control logic) over a managed one (the managed home) that can be independently designed and deployed. For example, Fig. 7 shows the OpenHAB dashboard where fire alarms are activated due to the high temperature values into the rooms of the first floor, which alert for a fire condition.

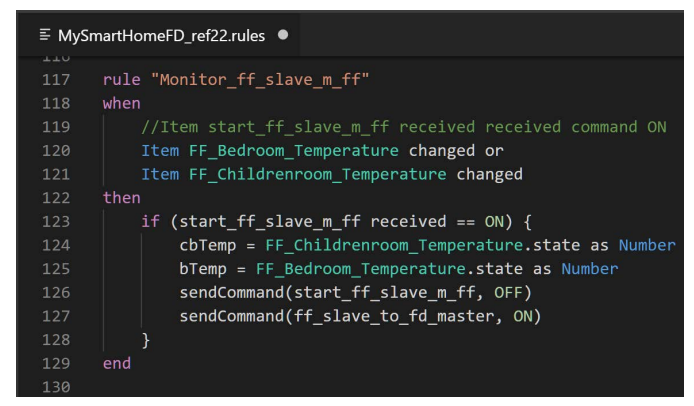

Figure 6: OpenHAB refined rule - Monitor_ff_slave_m_ff

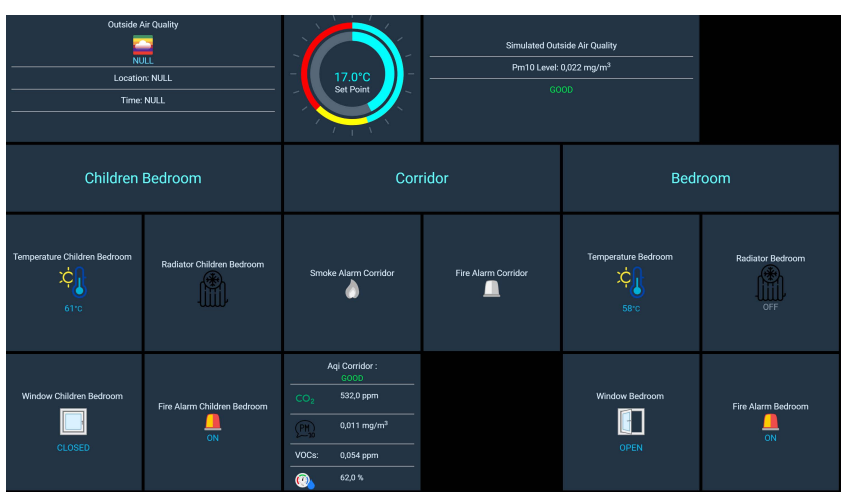

Figure 7: OpenHAB dashboard

\section{RELATED WORK}

We here review selected works that are related to the adoption of the MAPE-K feedback control loop model for smart-home solutions.

In [16], an autonomic trust management framework based on MAPE-K feedback control loop to evaluate the level of trust in a IoT cloud ecosystem is presented. The effectiveness of their framework is evaluated in a smart home environment; the IoT devices sense the trust parameters and exchange information to the trust agents virtualized in the cloud network.

In [5], an approach that enables the design of self-adaptive systems in a modular way is presented and evaluated through a use case based on a smart home scenario. The approach uses conventional GoF design patterns to separate the adaptation logic from the functional one and to facilitate the reuse of adaptation expertise at design phase. These patterns are used at three different layers of abstraction (the functional layer, the logical layer, and the technical one) for the modeling of the MAPE-K components.

ActivFORMS (Active FORmal Models for Self-adaptation) [9] is a virtual machine to realize self-adaptation with guarantee. Timed automata are used to design the MAPE-K loops accomplishing the adaptation goals. These models are verified off-line and at runtime, and can be updated on the fly to support changing goals. The approach has been validated on networked distributed system scenarios as networked smart-homes, multi-robot systems, and IoT Systems with mobile devices. Unlike our approach, it does not support coding of the models in a target implementation platform. 
The recent contribution in [17] presents a general framework in the context of cyber-physical systems for retrofitting existing (legacy) service-oriented workflow management engines with adaptation capabilities based on the MAPE-K control loop model. The approach is illustrated with the help of a simple scenario of smart home lighting process in OpenHAB, retrofitted via a feedback service call in order to provide constant room illumination.

The smart home gateway OpenLicht by Infineon [6] has an adaptive software architecture based on a single MAPE-K loop with an additional learner component to realize a self-learning lighting feature in OpenHAB. OpenHAB is mainly used as binding infrastructure for the hardware architecture and for the implementation of the Execute component of the OpenLicht system. The other components, including the knowledge, run as an extra home system (another node of the home network) and invoke remote services in a cloud-based server. Besides, no MAPE patterns are adopted.

With respect to the works mentioned above, by using the MSL framework we elevate MAPE-K loops to first-class entities for structuring the adaptation logic of any self-adaptive system in the early design phases and for fostering (in a broad sense) pattern-oriented modeling. Despite similarities and differences with our approach, all the works mentioned above can be used as back-end frameworks to complement and complete for different purposes the adaptation logic design started in MSL. Our approach can be also used as general framework for adding autonomous capabilities to legacy systems based on feedback loops and, therefore, for retrofitting existing home automation processes as well. Sensors from the legacy smart systems would provide information that is monitored and analyzed; decision and planning of adaptations would then performed by instrumenting the effectors of the legacy system.

\section{CONCLUSION AND FUTURE WORK}

We here conceived a control architecture style for smart home systems based on patterns of MAPE-K feedback loops to enforce desired adaptation goals and their interrelation. The decentralized recurring structures of MAPE loops devised in [19] are considered here as mainstream patterns of MAPE-K loops. Decentralization is conceptually realized by the notion of group of MAPE components. Groups of MAPE components can be designed, built, deployed and run on separate computational nodes (e.g., on different OpenHAB platform instances), and interact with other groups.

The adaptive control architecture model has been easily implemented and validated in a (virtual) smart home installation for the OpenHAB platform, thanks to the automatic transformation of an MSL model of a composite MAPE-K feedback loop for a home automation/adaptation concern of interest into automation rules of OpenHAB. Such direct encoding in the development board of the target platform is, in our opinion, one major advantage of our modelbased approach, since it avoids to introduce extra computational nodes (like PC desktops or other boards) in the home network.

As future direction, we intend to validate further the proposed architectural approach by developing demonstrators of a certain complexity, e.g., involving heterogeneous computational nodes of a home network infrastructure and/or smart buildings use case scenarios, and deal with, possibly, interfering MAPE-K loops for closely related home adaptation/automation concerns.

\section{ACKNOWLEDGMENTS}

P. Arcaini is supported by ERATO HASUO Metamathematics for Systems Design Project (No. JPMJER1603), JST. Funding Reference number: 10.13039/501100009024 ERATO. We thank Domenico Cimmino, Innovation Technology Manager of the Italian company Evolvere S.p.A., for sharing with us knowledge about the requirements and challenges of the smart home application domain.

\section{REFERENCES}

[1] Fahed Alkhabbas, Romina Spalazzese, and Paul Davidsson. 2017. Architecting Emergent Configurations in the Internet of Things. In 2017 IEEE International Conference on Software Architecture, ICSA 2017, Gothenburg, Sweden, April 3-7, 2017. IEEE Computer Society, 221-224.

[2] Paolo Arcaini, Raffaela Mirandola, Elvinia Riccobene, and Patrizia Scandurra. 2018. A DSL for MAPE Patterns Representation in Self-adapting Systems. In 12th European Conference on Software Architecture, ECSA 2018, Madrid, Spain, Sept. 24-28, 2018, Proceedings (LNCS), Vol. 11048. Springer, 3-19.

[3] Paolo Arcaini, Raffaela Mirandola, Elvinia Riccobene, and Patrizia Scandurra. 2019. A Pattern-Oriented Design Framework for Self-Adaptive Software Systems. In 2019 IEEE International Conference on Software Architecture Companion (ICSAC). 166-169.

[4] Paolo Arcaini, Elvinia Riccobene, and Patrizia Scandurra. 2017. Formal Design and Verification of Self-Adaptive Systems with Decentralized Control. ACM Trans. Auton. Adapt. Syst. 11, 4, Article 25 (Jan. 2017), 35 pages.

[5] Mohamed Lamine Berkane, Lionel Seinturier, and Mahmoud Boufaida. 2015. Using Variability Modelling and Design Patterns for Self-adaptive System Engineering: Application to Smart-home. Int. f. Web Eng. Technol. 10, 1 (May 2015), 65-93.

[6] K Bierzynski, F Kalleder, P Lutskov, F Rohde, DM Rodríguez, J Mena-Carrillo, R Schöne, and U Aßmann. 2018. OpenLicht - A Self-learning Lighting System based on OpenHAB. Smart Public Building 2018 (2018), 43.

[7] Sarah J. Darby. 2018. Smart technology in the home: time for more clarity. Building Research \& Information 46, 1 (2018), 140-147.

[8] Federico Quin et al. 2019. Efficient Analysis of Large Adaptation Spaces in Self-Adaptive Systems using Machine Learning. In Proc. of SEAMS 2019 - 14th International Symposium on Software Engineering for Adaptive and Self-Managing Systems.

[9] M. Usman Iftikhar and Danny Weyns. 2017. ActivFORMS: A Runtime Environment for Architecture-Based Adaptation with Guarantees. In 2017 IEEE International Conference on Software Architecture Workshops, ICSA Workshops 2017, Gothenburg, Sweden, April 5-7, 2017. 278-281.

[10] Pooyan Jamshidi, Javier Cámara, Bradley R. Schmerl, Christian Kästner, and David Garlan. 2019. Machine Learning Meets Quantitative Planning: Enabling SelfAdaptation in Autonomous Robots. CoRR abs/1903.03920 (2019). arXiv:1903.03920 http://arxiv.org/abs/1903.03920

[11] Jeffrey O. Kephart and David M. Chess. 2003. The Vision of Autonomic Computing. IEEE Computer 36, 1 (2003), 41-50.

[12] Ji Eun Kim, George Boulos, John Yackovich, Tassilo Barth, Christian Beckel, and Daniel Mossé. 2012. Seamless Integration of Heterogeneous Devices and Access Control in Smart Homes. In 2012 Eighth International Conference on Intelligent Environments, Guanajuato, Mexico, June 26-29, 2012. IEEE, 206-213.

[13] Christian Krupitzer, Felix Maximilian Roth, Sebastian VanSyckel, Gregor Schiele, and Christian Becker. 2015. A Survey on Engineering Approaches for Selfadaptive Systems. Pervasive Mob. Comput. 17, PB (Feb. 2015), 184-206.

[14] Mozilla 2019. Mozilla Web Thing API. https://iot.mozilla.org/wot/.

[15] MSL 2018. The MSL language. https://github.com/fmselab/msl.

[16] Suneth Namal, Hasindu Gamaarachchi, Gyu Myoung Lee, and Tai-Won Um. 2015. Autonomic trust management in cloud-based and highly dynamic IoT applications. In 2015 ITU Kaleidoscope: Trust in the Information Society, Barcelona, Spain, December 9-11, 2015. IEEE, 1-8.

[17] Ronny Seiger, Steffen Huber, Peter Heisig, and Uwe Aßmann. 2019. Toward a framework for self-adaptive workflows in cyber-physical systems. Software and System Modeling 18, 2 (2019), 1117-1134.

[18] W3C 2019. Web of Things (WoT) Architecture, W3C Candidate Recommendation 16 May 2019. https://www.w3.org/TR/2019/CR-wot-architecture-20190516/.

[19] Danny Weyns, Bradley R. Schmerl, Vincenzo Grassi, Sam Malek, Raffaela Mirandola, Christian Prehofer, Jochen Wuttke, Jesper Andersson, Holger Giese, and Karl M. Göschka. 2013. Software Engineering for Self-Adaptive Systems II: International Seminar, Dagstuhl Castle, Germany. Springer Berlin Heidelberg, Berlin, Heidelberg, Chapter On Patterns for Decentralized Control in Self-Adaptive Systems, 76-107. 